\title{
Optimisation of grasping object based on pressure sensor measurement for robotic hand gripper
}

\begin{abstract}
This paper presents an optimization of grasping object based on pressure sensor measurement. In this work, a commercial flexi force pressure sensors are assembled on the robotic hand gripper model with introducing new arrangement of pressure distributions. For optimization purpose, the authors introduced Wheatstone Bridge Circuit (WBC) methods whereby the pressure distributions were applied. Therefore, with the new arrangement of pressure sensor distribution it has promised higher sensitivity as well as stability for the developed robotic hand gripper system. This framework is considered as one of the techniques that can be applied for robotic hand applications based on pressure sensor especially for high-risk tasks such as military and dangerous environment. In other word, it can save people's life.
\end{abstract}

Keyword: Pressure sensor; Robotics; Wheatstone bridge circuit 